\title{
Physiotherapy or corticosteroid injection for shoulder pain?
}

\section{A W M van der Windt, L M Bouter}

\section{The choice is not clear cut and patients' expectations and preferences may affect the outcome}

n this issue Hay et al ${ }^{1}$ report the results of a randomised clinical trial evaluating the effectiveness of physiotherapy and corticosteroid injection for patients with unilateral shoulder pain. Shoulder pain is a common complaint; estimates of the annual incidence in general practice vary from 6.6 to 25 cases per 1000 patients. $^{2-4}$ Most patients are treated in primary care. If treatment with analgesics or non-steroidal antiinflammatory drugs (NSAIDs) is not successful, patients with persistent symptoms are often referred for physiotherapy or treated with local infiltration of a corticosteroid. ${ }^{4}$ Until recently, evidence on the effectiveness of these interventions was scarce, particularly for primary care patients. Over the past few years two randomised trials have been published that directly compared the effects of physiotherapy with corticosteroid injections. ${ }^{56}$ Both trials were carried out in Dutch general practice.

The trial by Hay et al adds important and relevant information to this existing evidence. The trial is characterised by a thorough design, enrolled a relatively large number of patients, used relevant outcome measures, and achieved a nearly complete six months' follow up of participants. When examining the results of these three primary care trials, three issues arise for discussion. Firstly, the short term findings are rather different, with the Dutch trials clearly showing better effects of corticosteroid injections, whereas the English trial reports similar outcomes for the two interventions. Secondly, all three trials show minor and non-significant differences at long term follow up. ${ }^{167}$ Thirdly, the somewhat ambiguous overall evidence may leave substantial room for considering patient preferences and expectations when applying the results in clinical practice.

\section{Inconsistent short term results:} differences in study group, content of treatment, or outcome measures? Figure la shows the self reported change of symptoms at 5-7 weeks of follow up for the three trials. As mentioned above, the short term differences strongly favour injections in the Dutch trials, whereas small, non-significant differences in the opposite direction are reported for the English trial. This heterogeneity in findings clearly exceeds random variation, which is confirmed by statistically testing for homogeneity $\left(\chi^{2}=32.4, p<0.00001\right)$. It is of interest to assess whether this heterogeneity can be explained by differences in clinically relevant factors, such as characteristics of the patient group or content of treatment. ${ }^{89}$ The trial by van der Windt $e t$ al specifically selected patients with restricted range of motion (painful and stiff shoulder), and reported slightly higher baseline scores for shoulder disability than the study by Hay et al. However, the three study groups are quite similar in age, sex, duration of symptoms, presence of additional neck pain, and severity of pain. Consequently, we consider it unlikely that differences in patient characteristics can explain the heterogeneity in study results.

More obvious differences can be noted about the content of treatment. In the trial of Hay et al a single injection of methylprednisolone was given, whereas injection treatment in both Dutch trials consisted of multiple (no more than three) injections of triamcinolone over a period of six weeks. Consequently, larger treatment effects may be expected in these two trials. However, fig la shows very consistent success rates for injection treatment: $73-76 \%$ of patients report improvement of symptoms. There is more variation across the trials in the perceived benefit from physiotherapy, with a relatively poor result in the trial by Winters et al $(20 \%)$, and a high success rate in the trial by Hay et al $(79 \%)$. However, there are, indeed, considerable differences in the physiotherapy given between the studies, as is discussed by Hay et al. Do these differences explain the different results for physiotherapy, and does this mean that the physiotherapy protocol used in the English trial is more effective?

There may be another explanation for the heterogeneity of results, related to the definition of outcome. All three studies evaluated self reported change, but used different scales. Winters et al used a dichotomous scale ("feeling cured" or not), whereas the two other trials used an ordinal five point or six point scale. The only difference between these latter scales is that van der Windt $e t$ al included one additional response

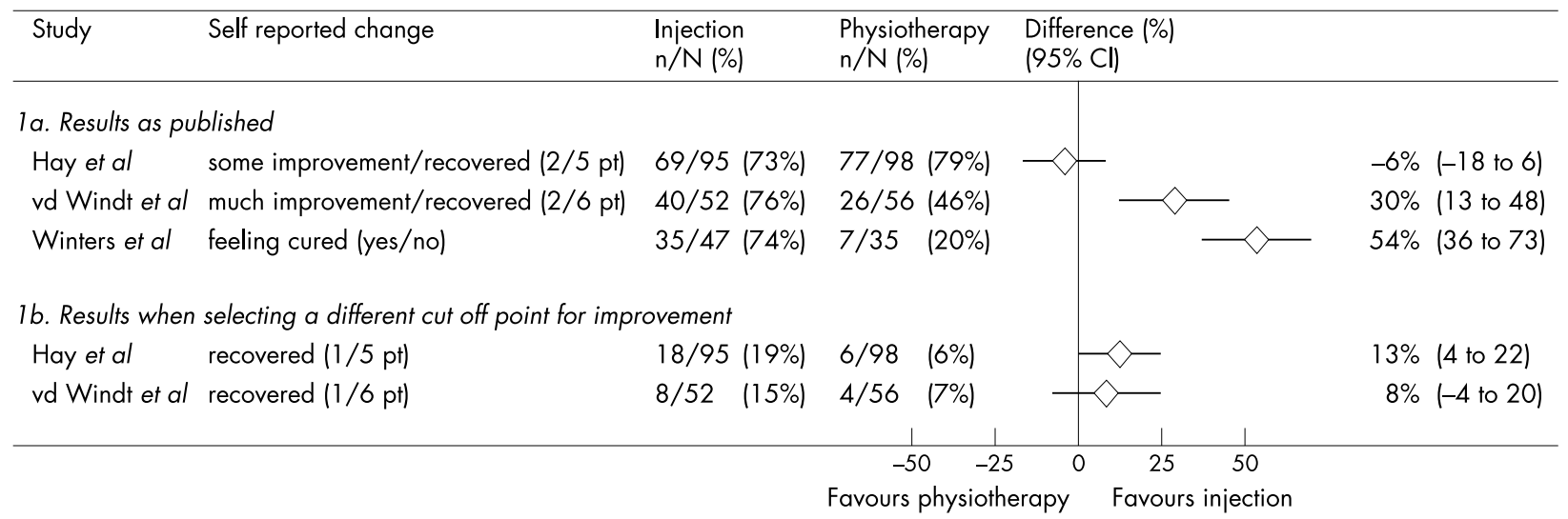

Figure 1 Results of three randomised trials comparing the effects of corticosteroid injections and physiotherapy on self reported change in patients with shoulder pain. ${ }^{150}$ 


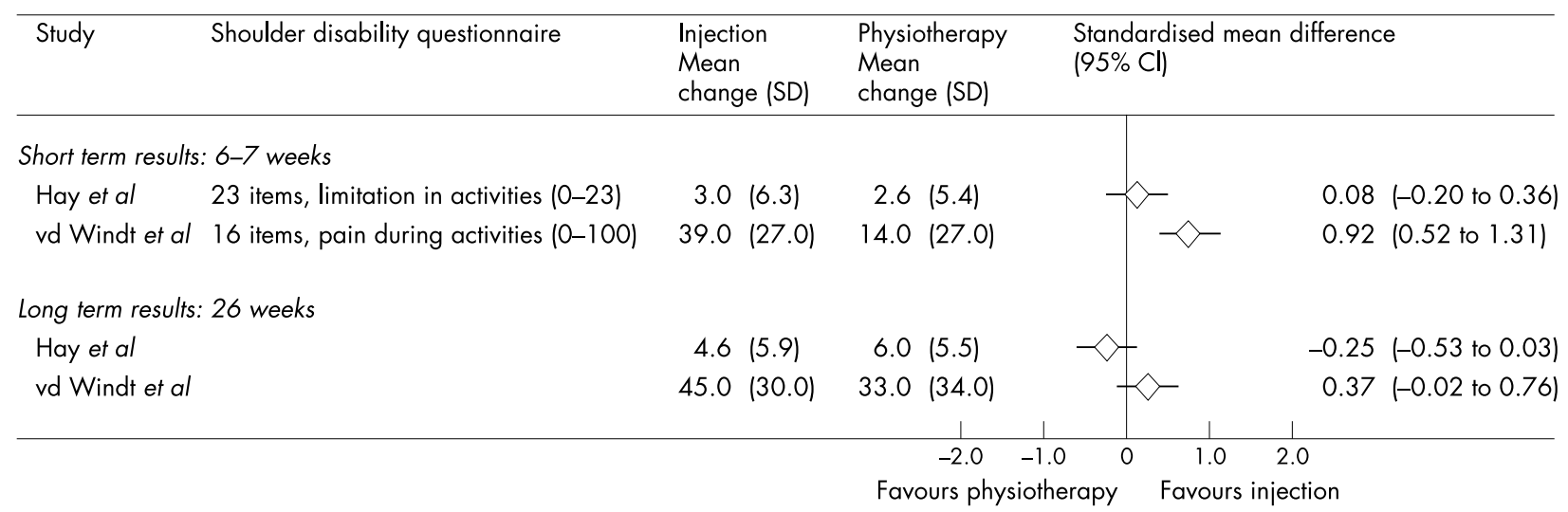

Figure 2 Results of two randomised trials comparing the effects of corticosteroid injections and physiotherapy on shoulder disability: short term and long term outcome. ${ }^{16}$

option-that is, "much improved" (second to complete recovery). This difference may seem trivial, but when reporting success rates, these scales are often dichotomised. The selection of a different cut off point may have considerable impact on the results of a trial, as fig $\mathrm{lb}$ shows. Of course, these results may be unstable owing to the small proportion of patients reporting full recovery, but conclusions would have been rather different if the researchers had decided on another definition of success.

In contrast with Hay et al, who reported the results for the entire scale, van der Windt et al only presented the dichotomised outcome. We can assure the reader that this cut off point was defined before the start of the trial, and already described in the grant proposal. Furthermore, it was based on previous research. ${ }^{10}$ None the less, the analyses presented in fig l strongly argue against the use of dichotomised outcomes without consensus among trialists about the definition of success or failure. Statistical techniques are available that enable the analysis of ordinal scales (for example, ordinal regression), which could be used in future analyses.

\section{"Consensus on a core set of outcome assessment measures is needed"}

Both trials also present the results for shoulder disability, based on self completed questionnaires. ${ }^{16}$ However, different questionnaires were used ${ }^{1112}$ with a different number of items, and different concepts being measured. A comparison of the results for shoulder disability can be facilitated by computing standardised mean differences (difference in mean change scores in the compared groups divided by their pooled standard deviation). ${ }^{13}$ The results (fig 2) confirm the better short term effect of corticosteroid injections in the Dutch study, and the lack of difference in the English trial.
Again, this might have been influenced by difference in the definition of outcome measures: the Dutch questionnaire strongly focuses on pain, whereas the English questionnaire evaluates limitations in activities (performance) in patients with shoulder pain.

\section{Lack of differences in long term outcome}

There is insufficient evidence about the long term effectiveness of injections or physiotherapy compared with a wait and see policy. ${ }^{14}{ }^{15}$ The three trials described here did not include such a control group. Small and non-significant differences were reported between injections and physiotherapy at 6-18 months' follow up, although the results presented by Hay et al seem to favour physiotherapy, particularly for shoulder disability and for the need for additional co-intervention during follow up. As yet, a cost effectiveness analysis has not yet been published for any of the three trials. The results of an economic evaluation will be of interest. If physiotherapy is successful in reducing medical consumption and (work) disability, as the results of Hay et al seem to suggest, these benefits may outweigh the additional costs of physiotherapy compared with injections.

In their paper Hay et al rightly argue that the results of the trials give patients and primary care clinicians a clear choice when deciding upon optimal treatment for shoulder problems. Short term benefits may be expected from corticosteroids (given the results of the Dutch trials) particularly for reduction of pain, but none of the treatments offer a clear clinical long term advantage. Other treatments are available to the general practitioner that might be of help in patients with shoulder pain, including NSAIDs, physical applications (for example, ultrasound, transcutaneous electrical stimulation), or manual treatment. Systematic reviews show that there is lack of evidence about the effectiveness of most interventions. ${ }^{14} 15$ Randomised placebo controlled trials have demonstrated better short term efficacy of NSAIDs, but there is no information about the benefit-risk ratio of NSAIDs compared with simple analgesics for shoulder pain. Furthermore, positive results have been reported for manual treatment as an addition to exercise therapy in patients with subacromial impingement syndrome, ${ }^{16}$ but this finding needs further confirmation. Several clinical guidelines for shoulder pain have been published. ${ }^{17-19}$ Given the ambiguous evidence, it is not surprising that some recommendations of guidelines are not consistent. The Dutch general practice guideline more strongly advocates the use of corticosteroid injections, when a wait and see policy or drug treatment has not been helpful, ${ }^{18}$ whereas the guideline issued by the American Academy of Orthopedic Surgeons recommends exercise programmes for most patients with shoulder pain. ${ }^{19}$

\section{Preferences and expectations}

Patients differ in the way they experience pain, and in the way they respond to treatment. Most patients will have expectations about treatment that may originate from previous experience, from the influence of media, family or friends, or from beliefs or worries. ${ }^{20}$ Such expectations may be expressed as preferences for a specific intervention in a trial.

\section{"Patients' expectations must be taken into account when trials are designed"}

Previous research has shown that expectations about treatment may influence treatment outcome..$^{21} 22$ In a randomised clinical trial comparing the effectiveness of acupuncture with massage in patients with low back pain Kalauokalani et al found greater improvement in disability scores in patients with higher expectations about the allocated treatment. ${ }^{21}$ 
Similar results were found in the trial by van der Windt et al. ${ }^{23}$

Being allocated to the preferred intervention influenced success rate, but only in patients allocated to injection treatment. These findings indicate that it is important to take patients' expectations into account when designing clinical trials, particularly where blinding is difficult or impossible, or in trials in which expectations or preferences may be stronger for one of the interventions under study. ${ }^{2324}$ Although evidence is not yet strong, clinicians should not ignore the potential influence of expectations and preferences about treatment in patients with musculoskeletal problems. Given uncertain or equal effects, as is the case in corticosteroid injection or physiotherapy for shoulder pain, it is advisable to explore and discuss any expectations and preferences with the patient.

\section{Conclusions}

Randomised clinical trials on the effectiveness of corticosteroid injections or physiotherapy for shoulder pain in primary care show inconsistent short term results. Differences in the content of treatment may explain these differences, but variation in selection and definition of outcome measures may also be of importance. Therefore, we agree with Hay et al that is important to obtain consensus on a core set of outcome measures for the assessment of shoulder pain, as has, for example, been proposed for low back pain research. ${ }^{25}$ Such a core set will enable comparison of results across studies, enhance the possibilities of secondary pooled analyses and of metaanalysis in systematic reviews, and facilitate the development of clinical practice guidelines.

Evidence for the long term outcome of physiotherapy or corticosteroid injections for shoulder pain is still limited. The long term effectiveness and cost effectiveness of both interventions need further investigation. Future research should also evaluate the potential influence of patient expectations and preferences on treatment outcome, particularly when few differences in (cost-) effectiveness are found, but strong differences in expectations across treatment options can be expected. This certainly holds for the treatment of shoulder pain in which interventions vary considerably in their burden on the patient, potential adverse reactions, and costs.

Ann Rheum Dis 2003;0:385-387

\section{Authors' affiliations}

\section{REFERENCES}

1 Hay EM, Thomas E, Paterson SM, Dzieczic K, Croft PR. A pragmatic randomised controlled trial of local corticosteroid injection and physiotherapy for the treatment of new episodes of unilateral shoulder pain in primary care. Ann Rheum Dis 2003:62:394-9.

2 Croft P. Soft tissue rheumatism. In: Silman AJ, Hochberg MC, eds. Epidemiology of the rheumatic diseases. Oxford: Oxford Medical Publications, 1993:375-421.

3 Lamberts H, Brouwer HJ, Mohrs J. Reason for encounter-, episode- and process-oriented standard output from transition project. Part I. Amsterdam: Department of General Practice/Family Medicine, University of Amsterdam, 1991.

4 Van der Windt DAWM, Koes BW, De Jong BA, Bouter LM. Shoulder disorders in general practice: incidence, patient characteristics and management. Ann Rheum Dis 1995:54:959-64

5 Winters JC, Sobel JS, Groenier KH, Arendzen HJ, Meyboom-de Jong B. Comparison of physiotherapy, manipulation, and corticosteroid injection for treating shoulder complaints in general practice: randomised, single blind study. BM 1997;314:1320-5

6 Van der Windt DAWM, Koes BW, Boeke AJP, Devillé W, De Jong BA, Bouter LM. Corticosteroid injections versus physiotherapy for painful stiff shoulder in primary care: randomised trial. BM 1998;317:1292-6.

7 Winters JC, Jorritsma W, Groenier KH, Sobel JS, Meyboom-de Jong B, Arendzen HJ. Treatment of shoulder complaints in general practice: long-term results of a randomised, single blind study comparing physiotherapy, manipulation, and corticosteroid injection. BM 1999;31:1395-6.

8 Thompson SG. Why sources of heterogeneity in meta-analysis should be investigated. BM 1994;309:1351-5.

9 Mulrow C, Langhorne P, Grimshaw J. Integrating heterogeneous pieces of evidence in systematic reviews. Ann Intern Med 1997; 127:989-95.

10 Van der Windt DAWM, Van der Heijden GJMG, Koes BW, De Winter AF, Devillé W, Bouter $L M$. The responsiveness of the shoulder disability questionnaire. Ann Rheum Dis 1998;57:82-7.
11 Croft P, Pope D, Zonca M, O'Neill T, Silman A. Measurement of shoulder related disability: results of a validation study. Ann Rheum Dis 1994;53:525-8.

12 Van der Heijden GJMG, Leffers P, Bouter LM. Development and responsiveness of the shoulder disability questionnaire. J Clin Epidemiol 2000;53:29-38.

13 Cohen J. Statistical power analysis for the behavioral sciences. 2nd ed. New Jersey: Lawrence Erlbaum Associates, 1988.

14 Green S, Buchbinder R, Glazier R, Forbes A. Systematic review of randomised controlled clinical trials of interventions for painful shoulder: selection criteria, outcome assessment, and efficacy. BM 1998;316:354-60.

15 Speed CA, Hazleman BL. Shoulder pain. In: Clinical evidence: a compendium of the best available evidence for effective health care. London: BM Publishing Group, 2000:issue 4.

16 Bang MD, Deyle GD. Comparison of supervised exercise with and without manual physical therapy for patients with shoulder impingement syndrome. J Orthop Sports Phys Ther 2000;30:126-37.

17 Philadelphia Panel. Evidence-based clinical practice guidelines on selected rehabilitation interventions for shoulder pain. Physical Therapy 2001;81:1719-30.

18 Winters JC, De Jongh AC, Van der Windt DAWM, Jonquière $M$, De Winter $A F$, Van der Heijden GJMG, et al. General practice guideline on shoulder complaints [NHG-Standaard Schouderklachten]. Huisarts \& Wetenschap 1999;52:222-31.

19 AAOS. Clinical guideline on shoulder pain. Rosemont, IL: American Academy of Orthopedic Surgeons, 2001

20 Till JE, Sutherland HJ, Meslin EM. Is there a role for preference assessment in research on quality of life in oncology? Qual Life Res 1992; 1:31-40.

21 Kalauokalani D, Cherkin DC, Sherman KJ, Koepsell TD, Deyo RA. Lessons from a trial of acupuncture and massage for low back pain. Patient expectations and treatment effects. Spine $2001 ; 26$ : 1418-24.

22 Jackson JL, Kroenke K. The effect of unmet expectations among adults presenting with physical symptoms. Ann Intern Med 2001;134:889-97.

23 Van der Windt DAWM, Van Aarst $M$ Heemskerk MAMB, Koes BW, Bouter LM. Practical aspects of conducting a randomised pragmatic trial in primary care: patient recruitment and outcome assessment. $\mathrm{Br} J$ Gen Pract 2000;50:371-4.

24 Lambert MF, Wood J. Incorporating patient preferences into randomised trials. J Clin Epidemiol 2000;53:163-6.

25 Deyo R, Battie M, Beurskens A, Bombardier $C$, Croft $P$, Koes B, et al. Outcome measures for low back pain research. A proposal for standardised use. Spine 1998;23:2003-13. 